\title{
Foscarnet as treatment for cytomegalovirus retinitis following bone marrow transplantation
}

\author{
P.S. Ganly, C. Arthur, J.M. Goldman and W.E. Schulenburg \\ Department of Haematology, Hammersmith Hospital, Du Cane Road, London W12 OHS, and Western \\ Ophthalmic Hospital, Marylebone Road, London NWI 5 YE, UK.
}

\begin{abstract}
Summary: This report describes a patient with chronic granulocytic leukaemia who developed cataracts on buśulphan treatment. Following allogeneic bone marrow transplantation, he developed cytomegalovirus retinitis, which was treated successfully with trisodium phosphonoformate (foscarnet). Cytomegalovirus retinitis and its therapy, and busulphan-induced cataract are discussed.
\end{abstract}

\section{Introduction}

Acquired retinitis caused by cytomegalovirus (CMV) infection is now seen more often because it occurs as a complication of acquired immunodeficiency syndrome (AIDS). Previously it was described mainly in patients immunosuppressed for renal transplantation or by chemotherapy. It has typical ophthalmological features of haemorrhagic vasculitis and the diagnosis is made when these are present in association with evidence of active CMV infection. ${ }^{1}$ As illustrated by this case report, the prognosis for the non-AIDS patient may be better than the severe and permanent visual effects, including blindness, which were common before specific anti-viral therapy was available.

\section{Case report}

A 23 year old Pakistani man was found to have Philadelphia chromosome positive chronic granulocytic leukaemia in 1976. He was treated intermittently with busulphan. He had received a total dose of $2.6 \mathrm{~g}$ by 1986 , when he underwent bone marrow transplantation from his HLA-identical sister. Both the patient and his bone marrow donor were CMV-positive by complement fixation tests. As pre-transplant conditioning he received daunorubicin, cyclophosphamide and total body irradiation. The donor bone marrow was depleted of T-cells in vitro.

Eighteen months prior to transplantation he had

Correspondence: P.S. Ganly, M.R.C.P., Department of Haematology, Hospital for Sick Children, Great Ormond Street, London WC1N 1EH, UK.

Accepted: 22 December 1987 noted blurring of vision in his right eye. His visual acuity was then right-6/6, left-6/6. (All readings given are corrected visual acuities.) $\mathrm{He}$ had bilateral posterior sub-capsular lens opacities. The fundi were normal.

Six weeks post-transplant he developed graftversus-host disease (GVHD) of the gut and skin, and was treated with methyl prednisolone. Six months post-transplant he still required corticosteroids to control chronic GVHD. Ophthalmological examination remained unchanged.

At 7 months post-transplant he noted further deterioration of vision in his right eye. A visual acuity of $6 / 18$ was recorded in the right eye and he maintained a visual acuity of $6 / 6$ in the left. There was no significant change in the lens opacity of either eye. On dilated fundoscopy a mid-peripheral haemorrhagic retinal vasculitis with necrosis, associated with vitreal inflammation was seen in the right eye. The appearance of the right fundus was characteristic of CMV retinitis. ${ }^{1}$ The left fundus was normal. CMV was isolated from the patient's urine.

Since retinal detachment was regarded as a possible complication, argon laser photocoagulation was applied through $360^{\circ}$ posterior to the necrotic retina. Methyl prednisolone was reduced from $16 \mathrm{mg}$ to $4 \mathrm{mg}$ daily. A continuous intravenous infusion of trisodium phosphonoformate (foscarnet) was commenced.

Initially his vision further deteriorated to counting fingers only on the right side. Two cotton wool spots developed at the left peri-macular area but did not affect his central vision. After 9 days of treatment the right fundus appeared less actively inflamed and there was no further progression of

(C) The Fellowship of Postgraduate Medicine, 1988 
necrosis. After 14 days his GVHD had significantly worsened, and he recommenced methyl prednisolone $40 \mathrm{mg}$ daily. He continued on a regimen of intermittent foscarnet; one third of the full dose was given by slow intravenous infusion five times weekly.

The retinitis and vitritis continued to resolve with progressive improvement of visual function in the right eye. Six weeks later there was a sharply defined junction between the normal and the infarcted retina. Islands of surviving retina were apparent within the infarcted retina. The cotton wool spots in the left fundus did not increase in number or size. Foscarnet was discontinued, after a total treatment time of 6 weeks.

Six months later the patient is now well. He still takes $5 \mathrm{mg}$ methyl prednisolone on alternate days. His visual acuity is right $-6 / 18$, left $-6 / 6$. The right fundus shows inactive retinal scarring; the left fundus is normal.

\section{Discussion}

The patient reported here developed CMV retinitis as a result of immunosuppression following allogeneic bone marrow transplantation. A single course of the antiviral drug foscarnet halted the progression of the retinitis while immunological recovery occurred. In addition this patient developed cataracts as a rare ophthalmological complication of busulphan treatment.

Evidence of prior infection by CMV is common in adults. Reactivation of CMV occurs in $57-96 \%$ of patients immunosuppressed due to organ or bone marrow transplantation ${ }^{2,3}$ or by chemotherapy. CMV retinitis as a manifestation of infection is well described in patients after renal or heart transplants, ${ }^{4}$ but has not apparently been reported previously in recipients of allogeneic bone marrow transplants. Immunosuppression in AIDS is associated with CMV retinitis in $34 \%$ of sufferers at autopsy. ${ }^{5}$

The natural history of acquired CMV retinitis is

\section{References}

1. Murray, H.W., Knox, D.L., Green, W.R. \& Sesel, R.M. Cytomegalovirus retinitis in adults. A manifestation of disseminated viral infection. $A m \mathrm{~J}$ Med 1977, 63: 574-584.

2. Fiala, M., Payne, J.E., Berne, T.V. et al. Epidemiology of cytomegalovirus after transplantation and immunosuppression. $J$ Infect Dis 1975, 132: 421433. variable. Occasionally it produces no visual symptoms, and is discovered on routine ophthalmoscopic examination. In these asymptomatic patients, retinitis may resolve spontaneously, if the immunosuppression can be reduced. ${ }^{4}$ When visual loss is marked, reflecting more extensive retinal involvement, progression is the rule, even if immunosuppression is decreased.

Therapy with specific anti-CMV drugs may be effective in the treatment of CMV retinitis in AIDS patients and others. Most experience has been gained with dihydroxy-propoxymethyl guanine (DHPG), an acyclic nucleoside analogue of guanosine which is structurally related to acyclovir. Since the initial case reports, ${ }^{6,7}$ others have confirmed that this drug can halt or even reverse the visual loss due to CMV retinitis; however, to prevent relapse in AIDS patients it must be administered in repeated courses, presumably reflecting the progressive impairment of cellmediated immunity in this population.

Foscarnet is another anti-CMV agent, which inhibits DNA polymerase in CMV, and is virostatic. ${ }^{8}$ There are two case reports of its use in the treatment of CMV retinitis, both in AIDS. One suggested that it might have produced a longer remission between treatment courses than DHPG, ${ }^{9}$ but the other did not confirm this and found it less effective than DHPG. ${ }^{10}$

Unlike the situation in AIDS, we believe our patient developed effective immunity to CMV following reactivation, because his retinitis did not relapse when treatment was stopped, and his immunosuppression was intensified to control resurgent GVHD.

Our patient already had a visual problem prior to the retinitis. In the absence of any other identifiable cause, the development of posterior subcapsular cataracts bilaterally pre-bone marrow transplant was ascribed to busulphan therapy. Although busulphan given in massive doses causes posterior subcapsular cataracts in rats, this complication has been described only rarely in patients receiving busulphan. ${ }^{11^{-13}}$ Cataract extractions are planned for this patient.

3. Meyers, J.D., Flournoy, N. \& Thomas, E.D. Cytomegalovirus infection and specific cell-mediated immunity after marrow transplant. J Infect Dis 1980 , 142: 816-824.

4. Pollard, R.B., Egbert, P.R., Gallagher, J.G. \& Merigan, T.C. Cytomegalovirus retinitis in immunosuppressed hosts. Ann Int Med 1980, 93: 655664. 
5. Pepose, J.S., Holland, G.N., Nestor, M.S., Cochran, A.J. \& Foos, R.Y. Acquired immunodeficiency syndrome; pathogenic mechanisms of ocular disease. Ophthalmology 1985, 92: 472-484.

6. Felsenstein, D., D'Amico, D., Hirsch, M.S. et al. Treatment of cytomegalovirus retinitis with 9-(2hydroxy-1-(hydroxymethyl) ethoxymethyl) guanine. Ann Int Med 1985, 103: 377-380.

7. Bach, M.C., Bagwell, S.P., Knapp, N.P., Davis, K.M. \& Hedstrom, P.S. 9-(1,3-dihydroxy-2-propoxymethyl) guanine for cytomegalovirus infections in patients with the acquired immunodeficiency syndrome. Ann Int Med 1985, 103: 381-382.

8. Editorial. Foscarnet. Lancet 1985, ii: 648-649.
9. Singer, D.R., Fallon, T.J., Schulenburg, W.E., Williams, G. \& Cohen, J. Foscarnet for cytomegalovirus retinitis. Ann Int Med 1985, 103: 962.

10. Acheson, J.F., Shah, S.M., Spalton, D.J., Bradbeer, C. \& Thin, R.N. Treatment of CMV retinitis in an AIDS patient. Br J Ophthalmol 1987, 71: 810-816.

11. Podos, S.M. \& Canellos, G.P. Lens changes in chronic granulocytic leukemia. Possible relationship to chemotherapy. Am J Ophthalmol 1969, 68: 500-504.

12. Ravindranathan, M.P., Paul, V.J. \& Kuriakose, E.T. Cataract after busulphan treatment. $B r$ Med J 1972: 1: 218-219.

13. Akman, N., Avanoglu, Y. \& Soysal, T. Cataract after busulphan therapy. Acta Haematol 1986, 76: 236. 